\title{
Rat vocalizations to shock: With and without a CS; in darkness and in light '
}

\author{
PAUL LEWIS', STEVE SUTER 3 , AND PIETRO BADIA \\ BOWLING GREEN STATE UNIVERSITY
}

Two experiments were performed to explore the effect of illumination on rat vocalizations to shock and to repeat a previous experiment in which the presence of a CS significantly reduced squealing to shock. The first experiment replicated the suppressive effect of a CS on vocalizations and indicated the effect may be obtained using onset or offset of illumination as the CS. Reduced squealing due to illumination alone was found in Experiment I using an independent group design but not found in Experiment II using a repeated measures design. A marked increase in vocalizations over trials was observed in both experiments.

Badia, Suter, \& Lewis (1966) reported an experiment in which one group of rats received signaled shock (CSUCS) and another group received the shock (UCS) alone. The dependent variable for both groups was the number of vocalizations elicited during a 2 sec UCS presentation. These investigators found the UCS elicited more vocalizations when presented alone than when it was preceded by a CS.

This UCR diminution was predicted from the theoretical statements of Perkins (1955). Perkins argues that conditioned responses which occur following CSUCS pairings prepare the organism for the UCS. This preparation either maximizes the reinforcing properties of appetitive stimulation or minimizes the aversiveness of noxious stimuli. The latter effect was the one predicted and confirmed.

Unconditioned response magnitude has also been studied by other investigators. Kimble \& Ost (1961) report observing gradually decreasing eyeblink UCRs during a classical conditioning experiment. Kimmel \& Pennypacker (1962) found the amount of diminution in the unconditioned GSR to relate to number of past CSUCS pairings. Interpretations of these findings has been in terms of Pavlov's (1927) concept of inhibition of delay (Kimmel, 1966).

In light of the limited information about the situation used in the Badia et al experiment, an interpretation without reference to the signaling properties of the CS appears tenable. The CS in the Badia et al study was an illumination increase resulting from the onset of a $6 \mathrm{~W}$ lamp. Since a delayed conditioning procedure was used for the CS-UCS group, the possibility exists that illumination alone may have suppressed vocalizing independent of other properties the CS may have had.

The present experiments were designed to test this possibility and to replicate the earlier experiment. Experiment 1

Method. The Ss were 24 naive Sprague-Dawley albino rats, 84 to 128 days of age, which were randomly assigned to one of four groups; half the Ss in each group were males. The four groups were CSOnset, CS Offset, UCS Light, and UCS Dark.

For the CS Onset group, the onset of a $6 \mathrm{~W}$ bulb $3 \mathrm{sec}$ before shock served as the CS. For the CS Offset group, the chamber was illuminated during the entire session except during shock and 3 sec preceding each shock. Thus the interstimulus interval was $3 \mathrm{sec}$, and the CS terminated with the UCS for both CS groups. The light was on during the entire experimental session for Group UCS Light but absent during the session for Group UCS Dark. The intertrial interval (ITI) was 60 sec and the UCS duration $2 \mathrm{sec}$. All four groups received 36 shock presentations.

The apparatus was a clear Plexiglas compartment 9 in. long, 6 in. wide, and 7.5 in. deep, with a stainless steel grid floor. The UCS was scrambled shock (.28 mA) provided by a Lehigh Valley Constant Current shock source. A microphone centered in the roof of the apparatus led to a tape recorder. Vocalizations were recorded at $7.5 \mathrm{in.} / \mathrm{sec}$ and counted by $\mathrm{E}$ at $3.75 \mathrm{in.} / \mathrm{sec}$.

Results. As may be seen in Fig. 1, there is a marked increase in vocalizations over trials for each of the four groups. The groups appear to reach asymptote at the same rate, with the UCS Dark group reaching a level considerably higher than the other three. As was found in the Badia et al (1966) study, both CS groups showed fewer squeals than their respective control groups. In addition, both groups (CS Onset and UCS Light) shocked

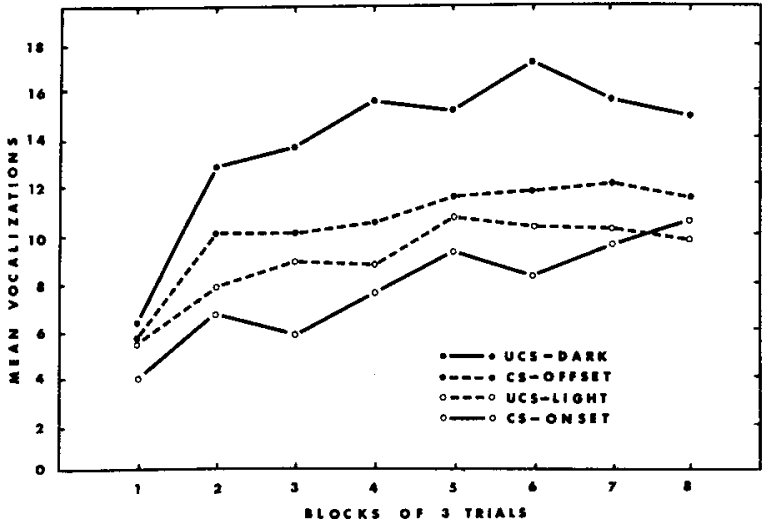

Fig. 1. Mean vocalizations to the UCS in blocks of three trials for four groups. The CS Onset group received CS-UCS trials, the CS was onset of illumination. The CS Offset group received CS-UCS trials, the CS was offset of illumination. The UCS Light and UCS Dark groups received only the UCS with the stimulus lamp on and off, respectively. 
in the presence of illumination vocalized less than those shocked in the dark.

Analysis of variance revealed a significant group effect $(F=3.10, \mathrm{df}=3 / 20, p<.05)$ and a significant trial block effect $(F=27.81$, df $=3 / 60, p<.005)$. The interaction effect was nonsignificant. Duncan's new multiple range test (Edwards, 1960, p. 136) indicated significant differences between UCS Dark and the remaining three groups and between the CS Light and CS Dark groups $(p<.05)$. These tests confirmed the group differences noted above, except for that between the CS Onset and UCS Light groups.

Discussion. The CS Onset and UCS Dark groups were identical to those in the Badia et al report and replicate the finding that an illumination CS suppresses rat squeals to shock. The significant difference between CS Offset and UCS Dark supports the interpretation that the suppression is independent of the nature of the CS (onset or offset). However, the significant difference between UCS Light and UCS Dark suggests that the presence of illumination alone may also be sufficient to suppress squealing.

Several other similarities are apparent between the present data and those reported by Badia et al. The present study also found gradually increasing UCS "acquisition" curves and no interaction between CS vs no CS treatments and trial blocks. This latter finding, plus the observation that both CS groups in this experiment tended to vocalize less on the first trial block, does not support explanations based on classically conditioned preparatory responses or gradually acquired conditioned inhibition. If efther were responsible for the suppression effect, it would not be expected to appear on the first trial and would be expected to change systematically over trials. If associative factors are ruled out, one must rely on some unconditioned effects of the CS to account for the attenuation in vocalizations.

It should be noted that no vocalizations to the CS were observed in any of the CS Ss. This finding is in contrast to Herbert (1946) and Cowles \& Pennington (1943), both of whom obtained reliable classical conditioning of rat vocalization responses. The lack of restraint in the present study stands out as a major difference between this study and the previous ones. Whether this is the critical variable remains to be determined.

\section{Experiment 2}

A second experiment was designed to further investigate the effect of illumination on rat vocalizations to shock. A repeated measures design appeared to be most appropriate since there was no indication of a "carry over" effect in Experiment 1. The use of a repeated measures design removed the possibility of non-random assignment in addition to adding statistical power. The elimination of possible non-random assignment is especially important since the differences between the UCS Light and UCS Dark groups in Experiment 1 may have resulted from this.

Method. Several procedural and apparatus changes were made between Experiments 1 and 2. The S's com- partment was placed in a ventilated wood-sand acoustical box (40 dB background level). Instead of tape recording each session and scoring the tapes, the vocalizations were displayed on a Tectronix (Model 564) storage oscilloscope and counted by $\mathrm{E}$ during the ITI (60 sec).

The Ss were eight female Sprague Dawley albino rats. All Ss received the same two conditions, 16 shocks with the stimulus lamp (6 W) on and 16 with the lamp off. The sequence of on-off trials was unsystematic except that no three trials in succession were alike, and four Ss received the sequence opposite the other four. When a trial required a change in illumination the change was made 3 sec after the preceding UCS. Thus on "light on" trials the light was either on during the entire ITI or else was turned on 55 sec before shock.

Results. Inspection of the data revealed nodifference in vocalizations between the light-on and light-off conditions. Ss vocalized an average of 8.93 times per trial in the presence of light and 8.85 times in its absence. Analysis of the effects due to illumination and to the interaction between illumination and trials were nonsignificant $(F<1.0)$; however, a significant trials effect was obtained ( $F=13.98, \mathrm{df}=6 / 42, \mathrm{p}<.001$ ).

Discussion. A significant increase in vocalizations as a function of trials was once again obtained. The lack of a difference between vocalizations in the presence and absence of illumination in Experiment 2 suggests the difference in Experiment 1 between the UCS Light and UCS Dark groups may have resulted from non-random assignment. The reduced number of vocalizations to the UCS when preceded by light CS (Badia et al) appears to be attributable to CSproperties rather than illumination. This interpretation is supported by current research in which an auditory CS (white noise) resulted in a similar suppression effect.

\section{References}

Badia, P., Suter, S., \& Lewis, P. Rat vocalizations to shock with and without a CS. Psychon. Sci., 1966, 4, 117-118.

Cowles, J. T., \& Pennington, L. A. An improved conditioning technique for determining auditory acuity of the rat. J. Psychol., $1943,15,42-47$.

Herbert, M. J. An improved technique for studying the conditioned squeak reaction in hooded rats. J. gen. Psychol., 1946, 34, 67-77.

Kimble, G. A., \& Ost, J. W. A conditioned inhibitory process in eyelid conditioning. J. exp. Psychol., 1961, 61, 150-156.

Kimmel, H. D., \& Pennypacker, H. W. Conditioned diminution of the UCR as a function of the number of reinforcements. J. exp. Psychol., 1962, 64, 20-23.

Kimmel, H. D. Inhibition of the unconditioned response in classical conditioning. Psychol. Rev., 1966, 73, 232-240.

Pavlov, I. P. Conditioned reflexes. London: Oxford University Press, 1927. (Transl. by G. V. Anrep.)

Perkins, C. C., Jr. The stimulus conditions which follow learned responses. Psychol. Rev., 1955, 62, 341-348.

\section{Notes}

1. This research was supported in part by USPHS Grant MH 1305401 to the third author. Portions of this paper were reported at the Midwest. Psychol. Assoc., Chicago, May, 1966.

2. The first author was supported by a PHS Fellowship MH-33, 936-01 during this research.

3. Now at Johns Hopkins University. 